\title{
Identification of a novel splice variant of the human musashi-1 gene
}

\author{
LIN MA $^{1 *}$, YATING SHAN ${ }^{1 *}$, HELIANG MA ${ }^{2 *}$, IHAB ELSHOURA $^{1}$, \\ MUHAMMAD NAFEES $^{3}$, KAIYONG YANG ${ }^{1}$ and WU YIN ${ }^{1}$ \\ ${ }^{1}$ State Key Laboratory of Pharmaceutical Biotechnology, College of Life Sciences, Nanjing University, Nanjing, \\ Jiangsu 210046; ${ }^{2}$ Department of Radiology, Jinan Central Hospital, Jinan, Shandong 250013; \\ ${ }^{3}$ State Key Laboratory of Coordination Chemistry, School of Chemistry and Chemical Engineering, \\ Nanjing University, Nanjing, Jiangsu 210046, P.R. China
}

Received October 4, 2017; Accepted February 28, 2018

DOI: $10.3892 / \mathrm{ol} .2018 .9300$

\begin{abstract}
Musashi-1 (Msi1) is an evolutionarily conserved RNA-binding protein that has been reported to be the key regulator in malignancies and with involvement in cancer stemness. In the present study, a novel Msil transcript variant generated by alternative splicing was identified and termed Msi1 variant 2. This variant was observed to be ubiquitously expressed in cancerous and non-cancerous cells compared with its wild-type variant, which is preferentially expressed in cancer cells. Notably, the expression levels of Msi1 variant 2 were inversely associated with the protein expression levels of Msil in various cancer cells. This naturally truncated variant contains 899 nucleotides and a skipping event of exons 3 and 4, which leads to the emergence of a premature TGA stop codon in exon 5. The present results also demonstrated that hypoxia increased the resistance of $\mathrm{H} 460$ cells to cisplatin by suppressing the exon 3 and 4 skipping event of Msi1. In summary, the present study identified a novel splice variant of Msi1 lacking two complete RNA recognition motifs, and revealed the role of exon 3 and 4 skipping of Msil pre-mRNA in regulating cisplatin resistance under hypoxia. These observations indicate that targeting Msil alternative splicing could represent a valuable strategy to repress Msil signaling in tumors overexpressing this RNA-binding protein.
\end{abstract}

\section{Introduction}

Gene expression is a differential process under precise control, and the alteration of gene expression via diverse biochemical

Correspondence to: Professor Wu Yin, State Key Laboratory of Pharmaceutical Biotechnology, College of Life Sciences, Nanjing University, 168 Xianlin Avenue, Nanjing, Jiangsu 210046, P.R. China E-mail: wyin_nju@163.com

${ }^{*}$ Contributed equally

Key words: alternative splicing, musashi-1, exon skipping mechanisms may influence the fate of the cells with negative consequences, leading to the emergence of serious diseases $(1,2)$. As a mechanism of gene regulation, alternative splicing acts at the post-transcriptional level by sequential addition of pre-mRNA transcripts, which results in the generation of various mature mRNAs (3-5). This mechanism serves an essential role in generating proteome diversity in malignancies through the production of splice variants involved in key oncogenic pathways and resistance to chemotherapeutic drugs (6). Thus, alternative splicing has been considered as a novel class of biomarker for the diagnosis of various types of cancer, providing informative, specific and accurate analysis for the study of cancer $(7,8)$.

Musashi-1 (Msi1) is an RNA-binding protein that negatively regulates genes at the post-transcriptional level by competing with eukaryotic translation initiation factor $4 \mathrm{G}$ (eIF4G) (9). In mammals, Msi1 has been described as a marker of adult stem cells and progenitor cells in the central nervous system by regulating Notch signaling (10). A plethora of research demonstrates that Msil binds with the 3'-Untranslated Region (UTR) of mRNA and regulates mRNA stability and the translation of proteins involved in essential oncogenic signaling pathways, and it is implicated in maintaining cancer stem cell populations and promoting diverse types of cancer (11-15).

The present study identified a novel splice transcript of Msi1, namely Msi1 variant 2, which consists of 13 exons. Exon 3 and 4 skipping led to the acquisition of a new in-frame TGA stop codon in exon 5, predicting a truncated product lacking two RNA recognition motif (RRM) domains. This exon-skipping event was confirmed using minigene plasmid, and could be detected as a major RNA splice form of Msi1 compared with its canonical transcript Msi1 in various cell lines. The present study further investigated the preliminary function of exon 3 and 4 skipping of Msil pre-mRNA in mediating hypoxia-induced cellular resistance to cisplatin.

\section{Materials and methods}

Cell culture. NSCLC cell lines, including H460, H1792, H1975, A549/DDP (16) and H1299, and one established 
primary NSCLC cell strain, Lac521 (17), were cultured in RPMI-1640 medium (Wisent Biotechnology, Nanjing, China) supplemented with $10 \%$ fetal bovine serum (FBS; Hyclone; GE Healthcare Life Sciences, Logan, UT, USA). The NSCLC A549 and SW1573 cell lines were cultured in Dulbecco's modified Eagle's medium (DMEM) (Wisent Biotechnology) supplemented with 10\% FBS. Human colorectal carcinoma (CRC) cell lines, including SW620, SW480, HT29, HCT116, Lovo and Caco-2, were cultured in DMEM supplemented with $10 \%$ FBS. Human leukemia cell lines, including Jurkat, HL-60, U937 [ATCC ${ }^{\circledast}$ CRL1593.2 ${ }^{\text {TM }}$; American Type Culture Collection (ATCC), Manassas, VA, USA], CCRF-CEM, K562 and THP-1, were cultured in RPMI-1640 medium supplemented with $10 \%$ FBS. Human hepatocellular carcinoma (HCC) cell lines, including SMMC7721 and BEL-7402, were cultured in DMEM supplemented with 10\% FBS. The human breast carcinoma MDA-MB-231 cell line was cultured in DMEM supplemented with $10 \%$ FBS. The human cervical squamous carcinoma HeLa cell line and the human osteosarcoma MG63 cell line were cultured in DMEM supplemented with $10 \%$ FBS. As non-cancerous cell lines, human normal bronchial epithelial cell lines, including BEAS-2B and HBE1, were cultured in RPMI-1640 medium supplemented with 10\% FBS. The human normal liver cell line HL-7702 (18) was cultured in RPMI-1640 medium supplemented with 10\% FBS. The cell line 293T (used for transfection and expression analysis) was cultured in DMEM supplemented with 10\% FBS. The human normal pancreatic ductal epithelial HPDE6-C7 cell line (19) was cultured in RPMI-1640 medium supplemented with 10\% FBS. The A549/DDP, HL-7702 and HPDE6-C7 cell lines were purchased from Shanghai Institute of Biochemistry and Cell Biology, Chinese Academy of Sciences (Shanghai, China), as mentioned in previous studies $(16,18,19)$. The remaining cell lines were obtained from the ATCC and were supplied by its corporate agent, Zhongyuan Ltd. (Beijing, China).

Cell lines. In the present study, the Human non-small cell lung carcinoma (NSCLC) H1792 cell line was used to amplify the full-length cDNA of Msi1 variants while the 293T cell line was used to Non-cancerous cells including BEAS-2B, HBE1, HPDE6-C7 and HL-7702 and cancerous cells including NSCLC, CRC, HCC and leukemia cell lines, in addition to Lac521 cells, were used to detect the endogenous skipping of exons 3 and 4 of Msil pre-mRNA. The 293T cell line was used to verify the expression of Msil constructs and to confirm the existence of exon 3 and 4 skipping by a minigene assay. The $\mathrm{H} 460$ cell line was used to investigate the role of musashi-1 in mediating chemoresistance under hypoxia condition.

Antibodies. A rabbit polyclonal antibody against Msi1 (cat. no. GTX101540) was purchased from GeneTex, Inc. (Irvine, CA, USA). Mouse monoclonal anti-GAPDH immunoglobulin G (IgG) (cat. no. sc-47724) and horseradish peroxidase (HRP)-conjugated secondary IgGs, including goat-anti-rabbit IgG (cat. no. sc-2004) and goat-anti-mouse IgG (cat. no. sc-2005), were purchased from Santa Cruz Biotechnology, Inc. (Dallas, TX, USA).

Construction of Msil minigene plasmid. Genomic DNA was extracted from the H1792 cell line using DNAiso Reagent
(Takara, Japan). Briefly, cells were scraped and homogenized using $1 \mathrm{ml}$ DNAiso Reagent. The supernatant was removed into a new tube following centrifugation $(12,000 \mathrm{x} \mathrm{g}$ for $10 \mathrm{~min}$ at $4^{\circ} \mathrm{C}$ ) and $500-\mu 11 / 2$ volumes of ethanol was added. The DNA pellet was collected into a new tube and washed with $75 \%$ ethanol twice. The exon 2-intron 2-exon 3-intron 3-exon 4-intron 4-exon 5 (E2-I2-E3-I3-E4-I4-E5) region of Msil pre-mRNA was amplified using he LA Taq polymerase (Takara, Japan) following amplification protocol: $94^{\circ} \mathrm{C}$ for $1 \mathrm{~min}, 60^{\circ} \mathrm{C}$, for $1 \mathrm{~min}, 72^{\circ} \mathrm{C}$ for $5 \mathrm{~min}$ followed by post-extension for $15 \mathrm{~min}$. Prior to amplification, the DNA template was pre-denatured at $98^{\circ} \mathrm{C}$ for $10 \mathrm{sec}$. The amplified fragment was digested with BamHI and HindIII and then ligated into the mammalian expression vector pRK5 with a FLAG tag sequence (The backbone plasmid was kindly provided by professor Zichun Hua in School of Life Sciences in Nanjing University).

Small interfering RNA (siRNA) transfections. For transient knockdown studies, $1 \times 10^{6} \mathrm{H} 460$ cells were transfected with NC control or Msi1-specific siRNAs supplied by Shanghai GenePharma Co., Ltd, (Shanghai, China). (100 nM) using Lipofectamine ${ }^{\circledR} 2000$ (Thermo Fisher Scientific, Inc., Waltham, MA, USA), according to the manufacturer's protocol. The sequences of the designed siRNAs were as follows: Sense, Msi1-1 5'-GUGGAGAAAGUGUGUGAAAUU-3' and Msi1-2 5'-GGGUUACCCAGGUUUCCAAGC-3'. To examine the effect of hypoxia on chemoresistance to cisplatin, H460 cells were transfected with Msi1-1 and Msi1-2 siRNA and then cultured under normoxia $\left(95 \% \mathrm{O}_{2}, 5 \% \mathrm{CO}_{2}\right)$ or hypoxia $\left(1 \% \mathrm{O}_{2}\right.$, $5 \% \mathrm{CO}_{2}, 94 \% \mathrm{~N}_{2}$ ). After $24 \mathrm{~h}$ of siRNA-mediated knockdown, cells were treated with $10 \mu \mathrm{g} / \mathrm{ml}$ cisplatin purchased from Sigma-Aldrich (Merck KGaA, Darmstadt, Germany) and maintained under the same conditions for an additional $24 \mathrm{~h}$.

Cell apoptosis assay. Cell apoptosis was measured by Annexin V-fluorescein isothiocyanate/propidium iodide double staining, according to the manufacturer's protocols (BioVision, Inc., Milpitas, CA, USA). A total of 10,000 cells were analyzed by flow cytometry. Data were analyzed using FlowJo 7.6.1 software (FlowJo LLC, Ashland, OR, USA).

Western blot analysis. Cells were lysed in ice-cold cell lysis buffer [50 mM Tris- $\mathrm{HCl}$ (pH 7.4), $250 \mathrm{mM} \mathrm{NaCl}, 50 \mathrm{mM} \mathrm{NaF}$, $5 \mathrm{mM}$ EDTA, $5 \mathrm{mM} \beta$-glycerophosphate, $1 \mathrm{mM}$ sodium vanadate, $1 \%$ Nonidet P-40 and complete protease inhibitor cocktail (Roche Diagnostics, Indianapolis, IN, USA). Upon centrifugation $\left(12,000 \mathrm{x}\right.$ g for $10 \mathrm{~min}$ at $\left.4^{\circ} \mathrm{C}\right)$, the protein concentration was determined using the Bradford method. Protein samples (40-60 $\mu \mathrm{g}$ ) were separated by $10 \%$ SDS-PAGE, and then transferred onto polyvinylidene difluoride membranes (EMD Millipore, Billerica, MA, USA), which were then blocked with $5 \% \mathrm{w} / \mathrm{v}$ non-fat powdered milk in TBS Tween-20 (TBST) for $1 \mathrm{~h}$ at room temperature $(0.5 \mathrm{M} \mathrm{NaCl}, 20 \mathrm{mM}$ Tris- $\mathrm{HCl}$ and $0.05 \% \mathrm{v} / \mathrm{v}$ Tween-20; $\mathrm{pH}$ 7.4). The membranes were subsequently incubated with primary antibodies, including anti-Msi1 IgG (1:1,000 dilution) or anti-GAPDH IgG (1:1,000 dilution), at $4^{\circ} \mathrm{C}$ overnight. Following three washes with TBS for $5 \mathrm{~min}$ each, the membranes were incubated with HRP-conjugated secondary antibodies (1:500) at room temperature for $2 \mathrm{~h}$. The 
blots were visualized upon incubation with the Amersham ECL start western blotting detection reagent (GE Healthcare Life Sciences, Little Chalfont, UK).

RNA extraction and reverse transcription-polymerase chain reaction $(R T-P C R)$. Total RNAs were isolated with TRIzol Reagent (Invitrogen; Thermo Fisher Scientific, Inc., Waltham, MA, USA) following the manufacturer's protocol. RT reactions were performed at $42^{\circ} \mathrm{C}$ for $1 \mathrm{~h}$ with oligo (dT) as a primer using the RevertAid RT Reverse Transcription kit (Fermentas; Thermo Fisher Scientific, Inc., Waltham, MA, USA). The sequences of the primers used for amplifying the full length of Msil (primer set A) are 5'-TTGGATCCATGG AGACTGACGCGCCCCAG-3' (forward) and 5'-TTAAGC TTTCAGTGGTACCCATTGGTGAAGGCT-3' (reverse). The PCR product was electrophoresed through a $0.8 \%$ agarose gel. The sequences of the primers used for detecting the exon 3-exon 4 skipping event (primer set B) are 5'-CAAGATGTT CATCGGGGGACTCAG-3' (forward) and 5'-CTTGGGCTG TGCTCGCCGAGGGAAG-3' (reverse). The sequences of primers for the reference control GAPDH are 5'-TGTCCC CACTGCCAACGTGTCA-3' (forward) and 5'-AGCGTC AAAGGTGGAGGAGTGGGT-3' (reverse). To confirm the existence of Msi1 variant 2, the FLAG tag was used as the forward primer, alongside the downstream reverse primer in primer set $\mathrm{B}$, in order to detect the exogenous exon 2-exon 5 skipping event using a minigene plasmid. The PCR protocol was as follows: $94^{\circ} \mathrm{C}$ for $30 \mathrm{sec}, 60^{\circ} \mathrm{C}$, for $45 \mathrm{sec}, 72^{\circ} \mathrm{C}$ for $30 \mathrm{sec}$ followed by post-extension for $5 \mathrm{~min}$. PCR products were electrophoresed through a $2 \%$ agarose gel. Each gel was stained with ethidium bromide for visualization under ultraviolet light.

Statistical analysis. Data are presented as the mean \pm standard deviation. $\mathrm{P}<0.05$ was considered to indicate a statistically significant difference. Multiple comparisons were performed with two-way analysis of variance followed by Bonferroni's post hoc test. For data analysis, GraphPad Prism 5.0 software for Windows was used (GraphPad Software, Inc., La Jolla, CA, USA).

\section{Results}

Amplification of RNA variants of Msil. The Msi1 RT-PCR amplification products in the NSCLC H1792 cell line were separated on a $0.8 \%$ agarose gel for analysis, and two bands of Msil were observed (Fig. 1A). We speculated that at least two variants of Msil could exist, and thus, each amplification product was subsequently cloned and sequenced. Unexpectedly, three additional variants besides the canonical Msi1 (1,089 bp) were observed. These new variants were sequenced, and the 1,056-, 889- and 1,064-bp products were identified as new variants of Msi1, termed variant 1, 2 and 3, respectively (Fig. 1B).

Protein translation analysis of novel Msil transcripts. Alignment of the amino acid sequences of these novel Msi1 transcripts demonstrated the presence of the premature stop codons TGA in exon 5 for variant 2 and TAA in exon 4 for variant 3 due to ribosomal frameshift, predicting two truncated open reading frames (ORFs) without the intact RRM domains (Fig. 1C, D). The three Msil variants and the canonical form were cloned into the mammalian expression vector pRK5, containing a FLAG tag. Then these plasmids were transfected into $293 \mathrm{~T}$ cells for $24 \mathrm{~h}$. The exogenous FLAG was used as the negative control. As demonstrated by western blotting in Fig. 1E, a band shift was observed between Msi1 variant 1 and its wild-type version due to the lack of 11 amino acids in the C-terminus of variant 1 compared with the canonical Msi1. However, Msi1 variants 2 and 3 could not be detected by western blotting due to the acquisition of the premature stop codons (Fig. 1E).

Exons 3 and 4 skipping in Msil variant 2 leads to the premature termination of Msil translation. In order to verify the site where the frameshift of the Msi1 transcript occurs, an incomplete exon 3 in Msi1 variant 3 and a skipping event of two exons in variant 2 were identified, which led to the existence of the premature stop codons TGA in variant 2 and TAA in variant 3 (Fig. 1F). Msi1 variant 2 contains exons 2 and 5, but lacks exons 3 and 4 , and the acquisition of the new in-frame stop codon TGA results in a truncated ORF lacking the complete RRM1 and the following domains (Fig. 2A). Next, a minigene plasmid comprising E2-I2-E3-I3-E4-I4-E5 was constructed and transfected into 293T cells. As revealed by RT-PCR analysis, exogenous transcripts E2-E3-E4-E5 and E2-E5 were observed, confirming the existence of exon 3 and 4 skipping of Msi1 pre-mRNA in human cells (Fig. 2B).

Msil variant 2 transcript is preferentially expressed in non-cancerous cells. The exon 3 and 4 skipping event in Msi1 variant 2 could be distinguished from wild-type Msi1 using RT-PCR primers specifically designed to flank the E2-E3-E4-E5 coding region of Msi1. The mRNA level of Msil variant 2 was observed to be universally expressed in various non-cancerous and cancerous cells, including primary NSCLC Lac521 cells (Fig. 2C). Thus, Msi1 variant 2 could be a major form of its splicing transcripts. While wild-type Msi1 was predominantly expressed in cancerous cell lines, including NSCLC, HCC, CRC and leukemia, Msi1 variant 2 was preferentially expressed in non-cancerous cell lines (Fig. 2C). In addition, the expression level of Msi1 variant 2 transcript inversely associated with the protein level of wild-type Msi1 in cancer cell lines (Fig. 2D).

Hypoxia-induced retention of exons 3 and 4 in Msil increases cisplatin resistance. Considering that Msi1 wild-type was preferentially expressed in cancer cells, the present study evaluated the contributing factors to exon 3 and 4 skipping of Msil pre-mRNA in the tumor microenvironment. As a salient feature of solid tumors (20), hypoxia $\left(1 \% \mathrm{O}_{2}, 5 \% \mathrm{CO}_{2}\right.$ and $\left.94 \% \mathrm{~N}_{2}\right)$ was observed to suppress exogenous exon 3 and 4 skipping of Msil pre-mRNA (Fig. 3A). Consistent with the aforementioned observation, endogenous detection of exon 3 and 4 skipping of Msi1 revealed that hypoxia induced the retention of exons 3 and 4 of Msi1, and upregulated Msi1 protein levels (Fig. 3B). Hypoxic tumors are often resistant to conventional cancer therapies. Thus, the present study examined the effect of hypoxia on the chemoresistance to cisplatin. With the upregulation of Msi1 protein 


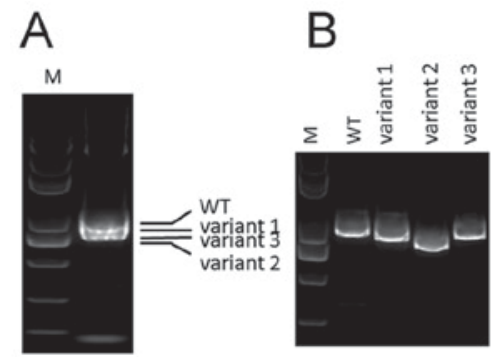

Variant 1

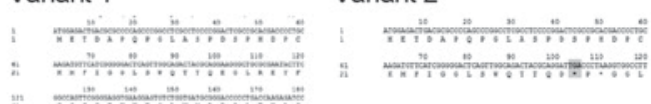

In

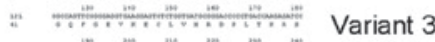

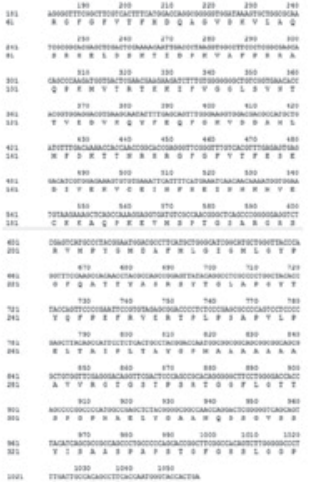

E

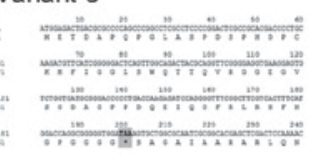

Variant 2

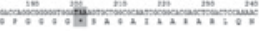

C

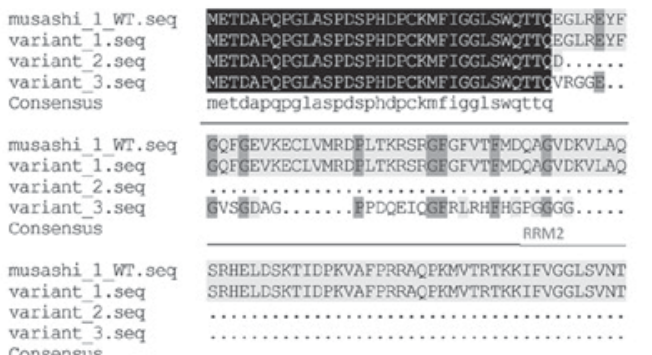

Consensus

musashi_1_WT.seq

variant_1.seq

variant 2. .seq

Consensus

musashi_1_wr.seq

variant_1.seq

variant ${ }^{-}$.. seq

variant $3 . \operatorname{seq}$

Consensus

musashi 1 WT.seq

variant 1 .seq

variant 2. seq

variant_-3.seq

Consensus

musashi_1 1 WT.seg

variant_1.seq

variant_2.seq

variant_3.seq

musashi 1 WT.seq

variant 1 - seq

variant-1.seq

variant 3. seq

Consensus

musashi_1_WT.seg

variant_1.seq

variant 2 .seq

variant $3 . \mathrm{se}$

Consensus

musashi_1_wr.se

variant_1.seq

variant_. 2. seq

TVEDVKQYFEQFGKVDDAMIMEDKTTNRHRGFGFVTFESE TVEDVROYFEOEGKVDDAMIMEDKTTNRHRGEGFVTFESE

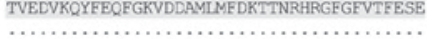

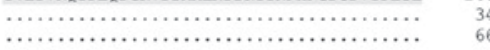

DIVEKVCEIHFHEINNKMVVECKKAQPKBVMSETGSARGRS DIVEKVCEIHFHE INNKMVECKKAQPKEVMSPTGSARGRS ......................................

YQFPEFRVERTPLPSAPVLPELTAIPLTAYGENANAMAA YQFPEFRVERTPLPSAPVLPELTAIPLTAYGEMAAAAAAA

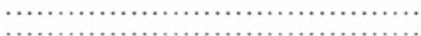

AVVRGTGSHPWIMAPPPGSTPSRTGGFLGTTSFGPMAELY AVVRGTG............. STPSRTGGFLGTTSFGPMAEL: .................................... ……

GAANQDSGVSSYISAASPAPSTGEGHSLGGPLIATAFTNG GAANQDSGVSSYISAASPAPSTGFGHSLGGPLIATAFTNG

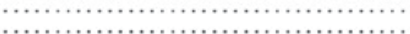
36

\section{F skipping region}

\begin{tabular}{|c|c|c|c|c|c|c|c|c|c|c|}
\hline \multirow[b]{2}{*}{ musashi $1 \mathrm{WT}$} & RT primer: & rimer $F$ & \multicolumn{3}{|c|}{ primer $R$} & & \multicolumn{4}{|c|}{ TGA } \\
\hline & exon 1 & exon 2 & exon 3 & exon 4 & exon 5 & exon 9 & exon 12 & exon 13 & exon 14 & exon 15 \\
\hline & & & & & & & & GTTCGAC. & TGA & \\
\hline \multirow[t]{2}{*}{ variant 1} & exon 1 & exon 2 & exon 3 & exon 4 & exon5 & exon 9 & exon 12 & exon 13 & exon 14 & exon 15 \\
\hline & & & E3-E. & pping & & & & GTTCGAC. & & \\
\hline \multirow[t]{2}{*}{ variant 2} & exon 1 & exon 2 & & & exon 5 & exon 9 & exon 12 & exon 13 & exon 14 & exon 15 \\
\hline & & & \multicolumn{3}{|c|}{ TTCGGG... IAA } & & & GITCGAC. & & \\
\hline variant 3 & exon 1 & exon 2 & exon 3 & exon 4 & exon 5 & exon 9 & exon 12 & exon 13 & exon 14 & exon 15 \\
\hline
\end{tabular}

Figure 1. Identification and translational analysis of Msi1 variants. (A) Amplification of Msi1 transcripts. (B) Cloning and separation of Msi1 variants. RT-PCR assays in (A) and (B) were performed using primer set A, as described in the Materials and methods. Bands were separated using $0.8 \%$ agarose gel electrophoresis. (C) Comparison of the amino acid alignment of the Msil canonical transcript and its variants. The predicted amino acid sequences of three Msi1 variants are aligned with that of the canonical transcript of Msi1. (D) Novel in-frame stop codons in Msi1 variants 2 and 3 . (E) Protein translation analysis of Msi1 variants. All Msi1 variants were cloned into the FLAG-tagged mammalian expression vector pRK5 and transfected into $293 \mathrm{~T}$ cells. Western blotting was performed following $8 \%$ SDS-PAGE. (F) Schematic representation of exon organization in Msi1 splice variants. The Msi1 transcript contains 15 exons, while variant 2 exhibits a skipping event of exons 3 and 4. Frameshift was observed in variants 2 and 3 with the existence of new in-frame stop codons (namely, TGA in variant 2 and TAA in variant 3). The arrows indicate the position of the RT-PCR primers used to detect the exon 3 and 4 skipping event. Msi1, musashi-1; M, marker; WT, wild-type; RT-PCR, reverse transcription-polymerase chain reaction; RRM, RNA recognition motif; F, forward; R, reverse; E, exon. 

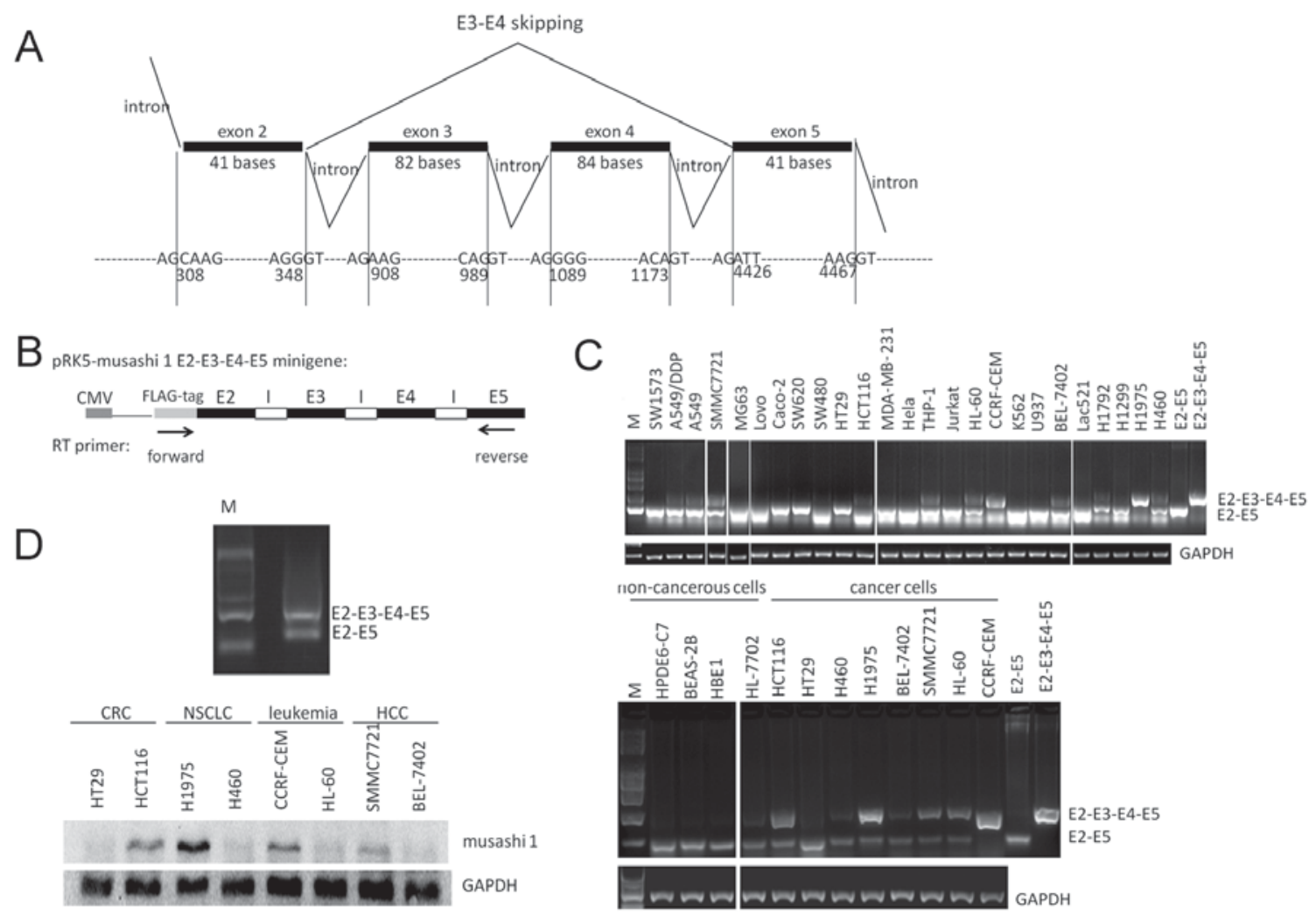

Figure 2. Detection of exon 3 and 4 skipping of Msi1 pre-mRNA. (A) Structure of the Msi1 spliceosome from exon 2 to exon 5. Exon 3 and 4 skipping of Msi1 pre-mRNA results in a sequence depletion of 166 nucleotides in Msi1 transcripts. (B) Detection of exogenous exon 3 and 4 skipping of Msi1 pre-mRNA using Msi1 E2-I2-E3-I3-E4-I4-E5 minigene plasmid. The arrows indicate the position of the RT-PCR primers used to detect minigene splicing. The exogenous exon skipping event of the Msil minigene plasmid was detected using a forward primer specific for the FLAG tag and a reverse primer from primer set B. (C) Detection of endogenous exon 3 and 4 skipping of Msil transcript in various cancer cells, including primary Lac521 cells, and NSCLC, CRC, leukemia, HCC and non-cancerous cell lines. Detection of exon 3 and 4 skipping was performed via RT-PCR assays using primer set B, as described in the Materials and methods. (D) Detection of Msil protein in various cancer cell lines. Msi1, musashi-1; E, exon; I, intron; CMV, cytomegalovirus; RT-PCR, reverse transcription-polymerase chain reaction; CRC, colorectal carcinoma; NSCLC, non-small cell lung carcinoma; HCC, hepatocellular carcinoma; M, marker.

level, hypoxia significantly increased cisplatin resistance in H460 cells, which was compromised in the absence of Msi1 (Fig. 3C). The results of western blotting confirmed the knockdown effect of Msi1 using the RNAi method (Fig. 3D). These observations indicate that hypoxia-induced cisplatin resistance requires downregulation of exons 3 and 4 skipping of Msi1 pre-mRNA.

\section{Discussion}

The present study identified a novel splice variant of Msi1, termed Msi1 variant 2, consisting of 13 exons. Msi1 has been identified as a marker of progenitor stem cells (21). Notably, Msi1 was also identified as a marker of CSCs $(22,23)$. The present study demonstrated that the skipping of exons 3 and 4 of Msi1 variant 2 led to the acquisition of a premature stop codon in exon 5 of the Msil transcript, resulting in the absence of complete RRM domains on the truncated Msi1 transcripts. Thus, this exon skipping event of Msil could be a way of negatively regulating cancer stemness stemness.

The biochemical mechanism by which Msi1 positively regulates cancer stemness or tumorigenesis is currently under investigation. Previous studies revealed that Msi1 suppresses genes such as Numb and p21 (Waf1) by competing with eIF4G $(9,10,24)$. These mRNAs are implicated in cell proliferation and protein modification, which are involved in tumorigenesis (25-27). However, the role of Msi1 in the progenitor stem cell compartment has been described only in a number of systems $(12,28)$. In the present study, the expression of Msi1 variant 2 was observed in various cancerous cell lines, including NSCLC, breast, colon and leukemia cell lines, as well as in several non-cancerous cell lines, suggesting that Msi1 variant 2 may be a broad-spectrum splice variant compared with its wild-type variant, which was preferentially expressed in malignant cells.

In addition, the Msi1 variant 2 does not contain two complete RRM domains, which are required for binding RNAs $(29,30)$. Although the present study has not demonstrated whether Msi1 and its variant 2 differ in their ability to interact with RNA, we anticipate that in the absence of RRM domains the function of Msi1 variant 2 may not be associated with the repression of translational initiation by competitively binding to Musashi-binding-element in the 3'-untranslated region of target transcripts. Thus, it is likely that Msi1 and its variant 2 may have different spectra of biological activity. The existence of distinct isoforms of Msi1 suggests that this gene may serve a complex role in regulating tumorigenesis, and elucidating the precise functions of its variants may provide further insights into the regulation of tumorigenesis and stemness. 
A
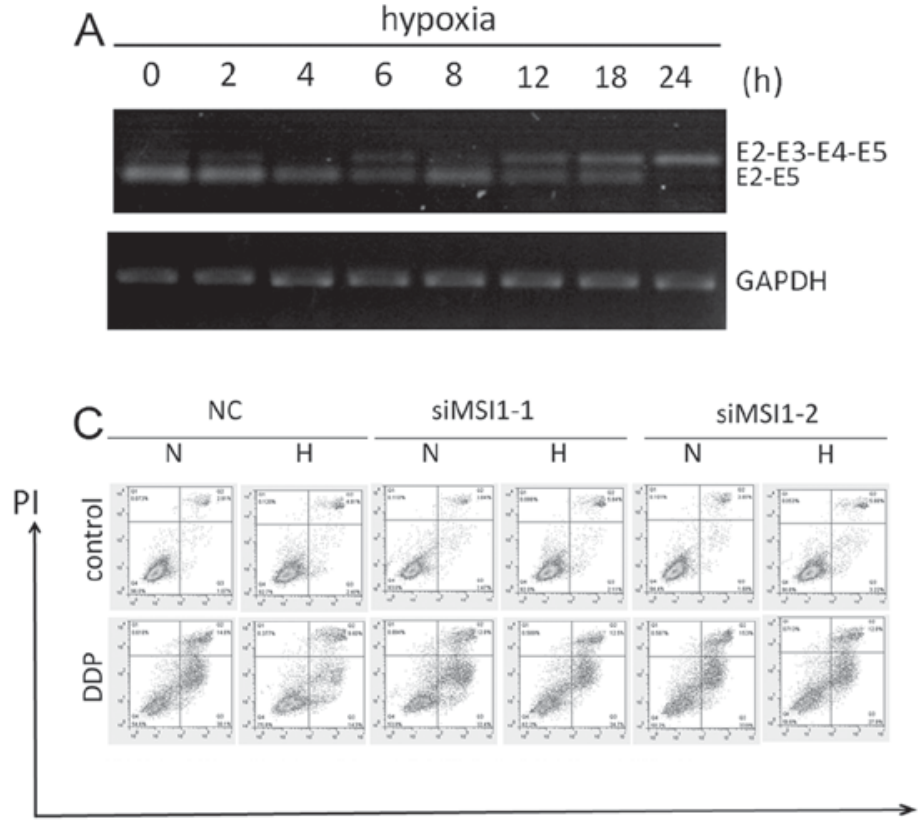

Annexin V-FITC
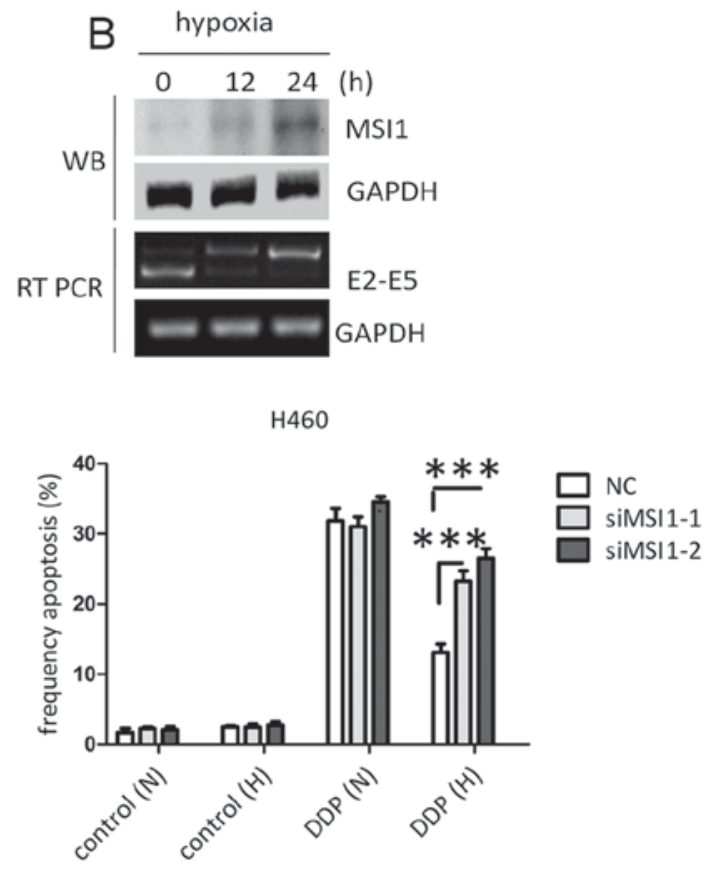

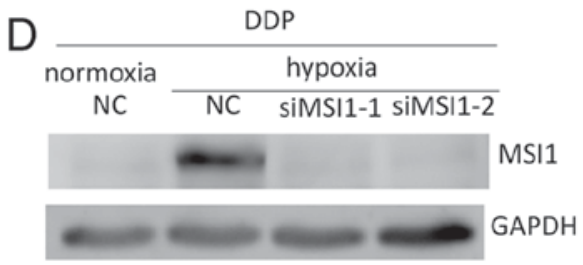

Figure 3. Hypoxia increases cisplatin resistance by promoting the retention of exons 3 and 4 of Msil pre-mRNA. (A) Effect of hypoxia on the exogenous exon 3 and 4 skipping event of Msi1 using Msi1 E2-I2-E3-I3-E4-I4-E5 minigene plasmid. (B) Effect of hypoxia on the exogenous exon 3 and 4 skipping event of Msi1 and its protein level. (C) Cisplatin-induced apoptosis in H460 cells under normoxia or hypoxia, in the presence or absence of Msil protein. (D) Knockdown efficiency of Msil protein. Data from triplicates are presented as the mean \pm standard deviation. ${ }^{* * *} \mathrm{P}<0.001$ (two-way analysis of variance). Msil, musashi-1; E, exon; I, intron; WB, western blotting; RT-PCR, reverse transcription-polymerase chain reaction; NC, negative control; si, small interfering; PI, propidium iodide; FITC, fluorescein isothiocyanate; N, normoxia; H, hypoxia; DDP, cisplatin.

An important strength of the current study is the association between the exon 3 and 4 skipping event of Msil and hypoxia-induced chemoresistance in NSCLC. Although solid tumors typically develop hostile microenvironments characterized by stress conditions, including irregular vascularization, and poor oxygen and nutrient supply, the cancer cells in these tumors exhibit unregulated growth under such hostile conditions through the activation of a series of survival pathways $(31,32)$. Under hypoxia, chemoresistance has been reported to be upregulated in various types of cancer $(33,34)$. Notably, Msil has been identified as an oncogene in several malignancies $(15,35)$. In the present study, hypoxia-induced cisplatin resistance in $\mathrm{H} 460$ cells required the retention of exons 3 and 4 in the Msil transcript, which frequently occurred in cancer cells compared with non-cancerous cells. Thus, the generation of Msil variant 2 by alternative splicing serves a tumor-suppressing role, and reversal of this exon skipping event of Msi1 under stress conditions may be responsible for tumorigenesis and malignant progression with increased chemoresistance.

Collectively, the present study achieved the following: i) Identified a novel untranslated isoform of Msil generated by skipping of exons 3 and 4; ii) evaluated its expression in various cell lines; and iii) preliminarily described the role of this exon skipping event in mediating chemoresistance. The aforementioned results indicate that the occurrence of the exon 3 and 4 skipping event in Msil could represent an important tool for malignancy diagnosis and therapy. With regard to therapeutic implications, targeting exon 3 and 4 skipping in Msil pre-mRNA could represent a valuable strategy to repress Msi1 signaling in tumors overexpressing this protein. However, there are several limitations affecting the present study. First, small molecules that could promote exon 3 and 4 skipping of Msil could not be identified due to the limited number of drugs tested. Second, the effect of exon 3 and 4 skipping of Msil on cancer stemness should be further investigated. In addition, future studies are required in order to clarify the exact role of Msil variant 2 in the development of malignancies.

\section{Acknowledgements}

The authors would like to thank Professor Zichun Hua (State Key Laboratory of Pharmaceutical Biotechnology, College of Life Sciences, Nanjing University) for providing the plasmid pRK5-FLAG. 


\section{Funding}

The present study was funded by the Natural Science Foundation of China (grant nos. 31071250, 81673462, 81473293, 91540119 and J1103521) and the Fundamental Research Funds for Central Universities via the Six Talent Peaks Project of Jiangsu (grant no. YY-012). The funders had no role in the study design, data collection, data analysis, decision to publish or preparation of the present manuscript.

\section{Availability of data and materials}

The datasets used and/or analyzed during the current study are available from the corresponding author on reasonable request.

\section{Authors' contributions}

WY, LM and HM designed the project. LM, YS, KY performed the experiments. HM, IE and MN analyzed the experimental data and offered discussion and suggestion. LM, YS, KY, IE and MN wrote the manuscript. WY and HM provided the financial support.

\section{Ethics approval and consent to participate}

The present article does not report any studies on human participants performed by any of the authors.

\section{Consent for publication}

Not applicable.

\section{Competing interests}

The authors declare that they have no conflicts of interest.

\section{References}

1. Rosenthal N: Regulation of gene expression. N Engl J Med 331: 931-933, 1994.

2. Krijger PH and De Laat W: Regulation of disease-associated gene expression in the 3D genome. Nat Rev Mol Cell Biol 17: 771-782, 2016.

3. Lopez AJ: Alternative splicing of pre-mRNA: Developmental consequences and mechanisms of regulation. Annu Rev Genet 32: 279-305, 1998.

4. Hong H, Braunschweig U, Gonatopoulos-Pournatzis T, Weatheritt RJ, Hirsch CL, Ha KCH, Radovani E, Nabeel-Shah S, Sterne-Weiler T, Wang J, et al: Multilayered control of alternative splicing regulatory networks by transcription factors. Mol Cell 65: 539-553.e7, 2017.

5. Talavera D, Orozco M and de la Cruz X: Alternative splicing of transcription factors' genes: Beyond the increase of proteome diversity. Comp Funct Genomics 2009: Article ID 905894, 2009.

6. Singh B and Eyras E: The role of alternative splicing in cancer. Transcription 8: 91-98, 2017.

7. Kim YJ and Kim HS: Alternative splicing and its impact as a cancer diagnostic marker. Genomics Inform 10: 74-80, 2017, 2012.

8. Hofstetter G, Berger AH, Slade N, Zorić A, Holzer B, Schuster E, Schuster E, Mobus VJ, Reimer D, Daxenbichler G, et al: Alternative splicing of p53 and p73: The novel p53 splice variant p53delta is an independent prognostic marker in ovarian cancer. Oncogene 29: 1997-2004, 2010.
9. Hironori K, Takao I, Hiroaki I, Masafumi T, Ken M and Hideyuki O: Neural RNA-binding protein Musashil inhibits translation initiation by competing with eIF4G for PABP. J Cell Biol 181: 639-653, 2008.

10. Imai T, Tokunaga A, Yoshida T, Hashimoto M, Mikoshiba K, Weinmaster G, Nakafuku $M$ and Okano $H$ : The neural RNA-binding protein Musashil translationally regulates mammalian numb gene expression by interacting with its mRNA. Mol Cell Biol 21: 3888-3900, 2001.

11. Chen HY, Lin LT, Wang ML, Lee SH, Tsai ML, Tsai CC, Liu WH, Chen TC, Yang YP, Lee YY, et al: Musashi-1 regulates AKT-derived IL-6 autocrinal/paracrinal malignancy and chemoresistance in glioblastoma. Oncotarget 7: 42485-42501, 2016.

12. Rezza A, Skah S, Roche C, Nadjar J, Samarut J and Plateroti M: The overexpression of the putative gut stem cell marker Musashi-1 induces tumorigenesis through Wnt and Notch activation. J Cell Sci 123: 3256-3265, 2010.

13. Toda M, Iizuka Y, Yu W, Imai T, Ikeda E, Yoshida K, Kawase T, Kawakami Y, Okano H and Uyemura K: Expression of the neural RNA-binding protein Musashi1 in human gliomas. Glia 34: 1-7, 2001.

14. Nishimoto $\mathrm{Y}$ and Okano $\mathrm{H}$ : New insight into cancer therapeutics: Induction of differentiation by regulating the Musashi/Numb/Notch pathway. Cell Res 20: 1083-1085, 2010.

15. Wang XY, Yu H, Linnoila RI, Li L, Li D, Mo B, Okano H, Penalva LO and Glazer RI: Musashil as a potential therapeutic target and diagnostic marker for lung cancer. Oncotarget 4: 739-750, 2013.

16. Shi $\mathrm{H}, \mathrm{Pu}$ J, Zhou XL, Ning YY and Bai C: Silencing long non-coding RNA ROR improves sensitivity of non-small-cell lung cancer to cisplatin resistance by inhibiting PI3K/Akt/mTOR signaling pathway. Tumour Biol 39, 2017. doi: $10.1177 / 1010428317697568$

17. Li X, You M, Liu YJ, Ma L, Jin PP, Zhou R, Zhang ZX, Hua B, Ji XJ, Cheng XY, et al: Reversal of the apoptotic resistance of non-small-cell lung carcinoma towards TRAIL by natural product toosendanin. Sci Rep 7: 42748, 2017.

18. Cui R, Zhang H, Guo X, Cui Q, Wang J and Dai J: Proteomic analysis of cell proliferation in a human hepatic cell line (HL-7702) induced by perfluorooctane sulfonate using iTRAQ. J Hazard Mater 299: 361-370, 2015.

19. Wan C, Gong C, Ji L, Liu X, Wang Y, Wang L, Shao M, Yang L, Fan S, Xiao Y, et al: NF45 overexpression is associated with poor prognosis and enhanced cell proliferation of pancreatic ductal adenocarcinoma. Mol Cell Biochem 410: 25-35, 2015.

20. Muz B, de la Puente P, Azab F and Azab AK: The role of hypoxia in cancer progression, angiogenesis, metastasis, and resistance to therapy. Hypoxia (Auckl) 3: 83-92, 2015.

21. Okano H, Kawahara H, Toriya M, Nakao K, Shibata S and Imai T: Function of RNA-binding protein Musashi-1 in stem cells. Exp Cell Res 306: 349-356, 2005.

22. Chiou GY, Yang TW, Huang CC, Tang CY, Yen JY, Tsai MC, Chen HY, Fadhilah N, Lin CC and Jong YJ: Musashi-1 promotes a cancer stem cell lineage and chemoresistance in colorectal cancer cells. Sci Rep 7: 2172, 2017.

23. Zhao W, Li Y and Zhang X: Stemness-related markers in cancer. Cancer Transl Med 3: 87-95, 2017.

24. Battelli C, Nikopoulos GN, Mitchell JG and Verdi JM: The RNA-binding protein Musashi-1 regulates neural development through the translational repression of p21WAF-1. Mol Cell Neurosci 31: 85-96, 2006.

25. Hong J, Liu Z, Zhu H, Zhang X, Liang Y, Yao S, Wang F, Xie X, Zhang B, Tan T, et al: The tumor suppressive role of NUMB isoform 1 in esophageal squamous cell carcinoma. Oncotarget 5: 5602-5614, 2014.

26. Kang Y, Ding M, Tian G, Guo H, Wan Y, Yao Z, Li B and Lin D: Overexpression of Numb suppresses tumor cell growth and enhances sensitivity to cisplatin in epithelioid malignant pleural mesothelioma. Oncol Rep 30: 313-319, 2013.

27. Chilosi M, Doglioni C, Magalini A, Inghirami G, Krampera M, Nadali G, Rahal D, Pedron S, Benedetti A, Scardoni M, et al: p21/WAF1 cyclin-kinase inhibitor expression in non-Hodgkin's lymphomas: A potential marker of p53 tumor-suppressor gene function. Blood 88: 4012-4020, 1996.

28. Wolfe AR, Ernlund A, Mcguinness W, Lehmann C, Carl K, Balmaceda N and Neufeld KL: Suppression of intestinal tumorigenesis in Apc mutant mice upon Musashi-1 deletion. J Cell Sci 130: 805-813, 2017.

29. Maris C, Dominguez C and Allain FH: The RNA recognition motif, a plastic RNA-binding platform to regulate post-transcriptional gene expression. FEBS J 272: 2118-2131, 2005. 
30. Pastore C, Topalidou I, Forouhar F, Yan AC, Levy M and Hunt JF: Crystal structure and RNA binding properties of the RNA recognition motif (RRM) and AlkB domains in human AlkB homolog 8 (ABH8), an enzyme catalyzing tRNA hypermodification. J Biol Chem 287: 2130-2143, 2012.

31. Giampietri C, Petrungaro S, Conti S, Facchiano A, Filippini A and Ziparo E: Cancer microenvironment and endoplasmic reticulum stress response. Mediators Inflamm 2015: 417281, 2015.

32. Urra H, Dufey E, Avril T, Chevet E and Hetz C: Endoplasmic reticulum stress and the hallmarks of cancer. Trends Cancer 2: 252-262, 2016

33. Sullivan R, Paré GC, Frederiksen LJ, Semenza GL and Graham $\mathrm{CH}$ : Hypoxia-induced resistance to anticancer drugs is associated with decreased senescence and requires hypoxia-inducible factor-1 activity. Mol Cancer Ther 7: 1961-1973, 2008.
34. Schnitzer SE, Weigert A, Zhou J and Brüne B: Hypoxia enhances sphingosine kinase 2 activity and provokes sphingosine-1-phosphate-mediated chemoresistance in A549 lung cancer cells. Mol Cancer Res 7: 393-401, 2009.

35. Lan L, Appelman C, Smith AR, Yu J, Larsen S, Marquez RT, Liu H, Wu X, Gao P, Roy A, et al: Natural product (-)-gossypol inhibits colon cancer cell growth by targeting RNA-binding protein Musashi-1. Mol Oncol 9: 1406-1420, 2015. 